\title{
Living alone in lockdown: Impact on mental health and coping mechanisms among young working adults
}

\author{
Tanya Raj $^{1}$ (D) Aishwarya Bajaj ${ }^{1}$ \\ Accepted: 16 February 2021 / Published online: 18 March 2021 \\ (C) The Author(s), under exclusive licence to Springer Science+Business Media, LLC, part of Springer Nature 2021
}

\begin{abstract}
In the wake of declaration of Covid-19 as a pandemic by the World Health Organization, the Prime Minister of India announced a nationwide lockdown to curb its spread. Subsequently, some groups of people found themselves away from their family or friends, and were unable to return to them. The aim of the research was to explore the lives of young adults who were living alone and working, during the lockdown in India. Focus was on their daily living, their relationships and their mental health. Twelve participants residing in various cities of India were included via the Internet. Focus group discussions were used to attain the objectives of the study. The data that emerged from the discussions, was analysed using thematic analysis. All participants had experienced different kinds of disruptions in their life due to the lockdown. This effect was mediated by their financial situation, their general comfort and preference for living alone, media, opportunities for non-virtual interactions and other kinds of recreation, quality of relationships and more. Though there were similarities in their coping methods, their perspective on the lockdown, the meaning they attributed to their current situation and their vision for their future was unique.
\end{abstract}

Keywords Lockdown $\cdot$ Living alone $\cdot$ Mental health $\cdot$ Coping mechanisms $\cdot$ Relationships $\cdot$ Daily living $\cdot$ Covid-19

\section{Introduction}

Covid-19 was declared a pandemic on 11th March 2020, by the World Health Organization (W.H.O, 2020). In India, due to the proliferation of the virus in March, the State declared a lockdown to enervate the spread of the virus ("PM Modi announces 21-day lockdown", 2020). Since the announcement of this lockdown was abrupt, multitudes of people found themselves in various unexpected circumstances. The lockdown created a plethora of financial and social repercussions on their life. It brought into focus psychological well-being and the impact of the crisis on it.

Among the vulnerable groups of humans affected, one of the groups were people who were living alone or were left alone in this lockdown. The usual sources of social and emotional support were cut off from them at a time of crisis. Plus, some of these people were also working from their homes. While on the one hand, these happened to be fortunate people to continue to hold on to their jobs at a financially unstable

Tanya Raj

1 New Delhi, India time, there were other quagmires to navigate with virtual workspaces. This is the group the researchers were interested in and wished to know more about.

A prerequisite to exploration of the lived experiences of this group is mapping their context. Research has highlighted the miscellaneous faces of stress individuals have weathered during the lockdown. But, the positive elements of this time have been documented, too. The role of the media and the lockdown regulations have been applauded as well as criticised. Some scholars have suggested methods of coping with the stress induced by the pandemic.

Stress as a Consequence of Lockdown Research has demonstrated that the uncertainty surrounding the lockdown and even about the post-lockdown scenario has created considerable stress, especially chronophobia which is the fear of time running out, and consequently, emotional preparedness is requisite to deal with this crisis (Benerjee, 2020; Naguy, Moodliar-Rensburg, \& Alamiri, 2020). Cellini, Canale, Mioni, and Costa (2020) elucidated that due to the lockdown, keeping track of time became intractable for young adults. They experienced boredom often and felt stuck. Not only this, a change in their sleeping habits was observed, with people going to sleep and waking up later than their usual routines, spending increased time in bed and reporting a poorer sleep 
quality. Their use of digital media had also increased. The researchers linked these changes to depression and anxiety in young adults. They explained the change in sleep cycle and habits with the lack of/decreased physical movement as well as reduced social zeitgebers like work schedules and social activities. Factors that have been identified as correlates of depression and PTSD include being female, low SES, interpersonal conflicts, high social media use, low social support and resilience (Torales, Higgins, Castaldelli-Maia, \& Ventriglio, 2020).

In addition to the sleep cycles, lockdown has an impact on daily routines and leads to limitations of personal freedom (Flesia, Fietta, Colicino, Segatto, \& Monaro, 2020). This is particularly true for the younger population because the older population find it easier to follow the restrictive measures.

Another factor linked to stress is risk perception, that is, an individual's belief in their susceptibility to the virus. Moreover, the physical distancing guidelines, for safeguarding oneself against the virus, give birth to social isolation that, in turn, contributes to stress, anxiety and depression (Holmes et al., 2020; Rajkumar, 2020). Social isolation may also result in social disconnection, lack of meaning, bereavement, entrapment, loss, feeling a burden, relationship breakdown. Besides, these studies demonstrated that the fear and panic can result in people hoarding resources. This is related to health anxiety, which is a consequence of erroneous interpretation of perceived bodily changes. In a pandemic situation, exacerbated by unreliable information by the media, health anxiety can become excessive, resulting in hoarding items, frequent medical consultation, avoidance of necessary health behaviour, lack of trust in public authorities and scapegoating specific sections of the population.

One more crucial source of stress is financial difficulties (De Sousa, Mohandas, \& Javed, 2020). Majority of India's population is engaged in the unorganized sector, and hence, do not have safety nets. In case of a protracted lockdown and pandemic, financial distress is expected to rise.

Silver Lining of the Lockdown Peshave and Peshave (2020) have reported that the lockdown has resulted in reduced pollution, increased attention towards sanitation and hygiene and decrease in financial expenditure. People have reported that they have received increased social support from friends and family, and they share more about their feelings with their family and friends (Zhang \& Ma, 2020). Citizens of India have been found to be spending their time during the lockdown in performing household chores with other family members, bonding with their family and on personal and work hygiene (Peshave \& Peshave, 2020). With respect to relationships with people one does not reside with, post declaration of need for social/spatial distancing, people are finding new ways to sustain their connections with each other (Abel \&
McQueen, 2020). People are spending more time to rest, relax and exercise (Zhang \& Ma, 2020).

However, kindness to those outside our close knit social circles is apparently not so common because instances of stigmatisation and discrimination against individuals from north east India, health care workers, flight attendants and others who have a foreign travel history have surfaced (Ganguly, Misra, \& Goli, 2020).

Lockdown Regulations and the Role of Media in a Pandemic There are reports of majority of Indian people on Twitter, applauding the decision of the lockdown (Barkur, Vibha, \& Kamath, 2020). Some researchers have indicated that the media needs to review its responsibility in creating panic instead of awareness (Goyal, Chauhan, Chhikara, Gupta, \& Singh, 2020; Hiremath, Suhas Kowshik, Manjunath, \& Shettar, 2020). Frequent use of media and reliance on unreliable sources of information has been found to be positively correlated with stress (Flesia et al., 2020). A probable reason is that the media often employs risk elevating messages which can give birth to anxiety, which in turn increases the tendency to consume more media, thus turning into a vicious cycle (Holmes et al., 2020). An ideal way of reporting the pandemic would be to rely on authoritative sources, minimise any graphic content and correct any misinformation or rumours. Torales et al. (2020) behove authorities to regularly update citizens about the crisis so that a sense of calm can be maintained because otherwise citizens look at other avenues for information like social media and consequently, they receive conflicting messages.

According to credible reports from the Lancet (2020), India acted swiftly by imposing a nationwide lockdown in March, as soon as Covid-19 cases in the country began their upward journey. Since physical distancing has been regarded as a necessary (WHO, 2020) precaution against the virus, a lockdown ensured restricted mobility of people by temporarily terminating the non-virtual functioning of all commercial workspaces, closing down of educational institutions as well as of places that attracted huge crowds such as temples and mosques. The objective was to curb the spread of the virus by ensuring physical distancing and by preparing healthcare infrastructure to deal with the infection once the lockdown was lifted. The Lancet further reports that despite its effectiveness, the lockdown hit roadblocks due to unequal access to healthcare, social and economic inequalities and diverse cultural values. Their report critiques the suddenness of the lockdown that led to problems like the mass exodus of lower class migrant workers.

Coping with the Pandemic Maintaining social connections, journaling, making \& sticking to routines, doing yoga and engaging in a pleasurable activity can help us to manage anxiety, depression and loneliness, along with measures taken by 
the government such as a website which provides accurate information about Covid-19, that busts myths, helplines, financial relief in terms of EMI (Hiremath et al., 2020). Inclusion of mental health care in disaster management plans has been accorded importance (Rajkumar, 2020).

The aim of this research was to explore the lives of young adults who were residing alone during the lockdown imposed due to Covid-19 pandemic, in India. The objectives of the present research are as following:

- Understanding of their present situation along with their activities of daily living with their role functions

- Understanding of their work from home and its management in the present situation with daily activities and role functions.

- Understanding the nature of human interactions and its effect on interpersonal functioning.

- Understanding the effect of present situations on their mental health.

- Understanding their coping mechanism in the present situation.

\section{Methodology}

To explore the lived experiences of young adults who were residing alone during the lockdown imposed due to Covid-19 pandemic, in India, an exploratory, qualitative, phenomenological research design was adopted (Denzin \& Lincoln, 2017). The phenomenon that was under inspection was the act of living alone during a lockdown. The aim was to elicit narratives about how the daily lifestyle and particularly the relationships of these individuals were impacted by the lockdown. Since the literature review is indicative of repercussions of the lockdown on mental health, mental well-being of participants was a requisite area of inquiry. In other words, the purpose of this study was to seek to capture and describe the essence of what it meant for individuals, residing alone and working to earn a living, to live through a lockdown, in India, imposed due to a pandemic. The researchers believe in the subjectivity of the human experience and the influence of context one is located in, on that experience. They were keen on exploring the influence of the larger context of a lockdown as well as the more specific immediate contextual setting of solitary living and working, on the participants, along with the perspectives of their individual meaning making process and coping mechanisms.

Sample Participants were chosen via purposive sampling. The inclusion criteria was an Indian citizen in the age range of 23 to 30 years, working and living alone during the lockdown imposed in March, April and May 2020, in
India. Information about the study was circulated on various social media platforms and individuals who were interested in the same as well as met the inclusion criteria, were included as participants. The focus group discussions were conducted when a minimum of 40 days had passed since the declaration of the first lockdown. It was acknowledged that the participants were in a vulnerable situation and the discussions were meant to explore their current experience. Therefore, potential participants were enquired about any history of mental or physical disturbance, to screen participants.

Method The primary tool of data collection was a focus group discussion, because the researchers wished to provide participants ample space to share their thoughts and feelings. Instead of individually interviewing young adults who were working and living alone, the researchers envisioned bringing the participants in the same space and encouraging them to share their experiences in a supportive environment. The intention was to facilitate a discussion between the participants who were in a similar setting. A focus group discussion guide was formulated on the basis of literature review and objectives of the research. Information about the research and an informed consent form were circulated via the internet. The participants who met the inclusion criteria and consented to participate in the research were emailed about the details of a virtual focus group discussion. A total of twelve participants were a part of the research. The discussion was facilitated by the researchers using a self-constructed guide. The virtual discussion was recorded, with permission of the participants, and transcribed. The transcriptions were analysed using thematic analysis. Three FGDs were conducted in this manner.

Measure Used The Self Constructed Guide was constructed on the basis of literature review and objectives of the research. The guide was categorized under following sections:

Understanding of their Present Situation along with their Activities of Daily Living with their Role Functions This section included questions around their present situation and differences from the situation before lockdown. This was followed with understanding their daily living activities and its management considering living alone.

\section{Understanding of their Work from Home and its Management} in the Present Situation with Daily Activities and Role Functions This section included questions around their experience of work from home and the effect of the present situation on their style of working, work quality and work life. This further included questions around their strategies to make their work more effective and its management with their daily activities and role functioning. 
Understanding the Nature of Human Interactions and its Effect on Interpersonal Functioning This section included their present experience and nature of human interactions, both virtual and non-virtual human interaction. This was followed with the impact of present situations on their human interactions and their meanings of these impacts. This also included understanding the impact of these human interactions on their social relationships and interpersonal functioning.

Understanding the Effect of Present Situations on their Mental Health This section included understanding of the effect of all the above on their mental health. This consisted of the effect of living alone, management of their work life with their daily activities and role functions, increase of virtual interactions and absence of human connectivity on their mental health. This also included understanding of their management of their biological functions such as physical health and fitness and sleep hygiene and its effect on their mental health.

\section{Understanding their Coping Mechanism in the Present}

Situation This section included understanding their approaches towards coping in the present situation. This further included their coping strategies and its effect on the present situation, as well as the differences between the present coping strategies and usual coping strategies before lockdown and pandemic. This was further concluded with their take away from the present time as their strength.

Thematic Analysis Thematic analysis was used in the present study to interpret the FGD transcripts. Thematic analysis was done by the authors themselves, as a unit, using the model of thematic analysis proposed by Clarke and Braun (2013). Both the authors did the initial coding of the transcripts at individual level first.This was followed with organizing all the codes into broader themes. This step was done collectively as a unit by both the authors. Once the preliminary themes were decided, it was reviewed. The process of reviewing included ensuring coherence and distinction among the identified themes. The differences in the reviewing process was resolved with an understanding of the contribution of the theme in the context of the whole data towards the objectives of the present study. During this process themes were refined further into main themes and sub themes as in context with the whole data set. Finally, all the themes were defined and their relationship between main themes and sub themes were established into a thematic model.

Ethical guidelines for psychological research were followed. Participants were informed about the major topics which will be covered in the discussion to allow them to mentally prepare themselves. The discussion was started with a basic mood check in. The discussion ended on the topic of coping and self-care. During the discussion, care was taken that no participant was judged for her/his narrative. It was ensured that no intrusive probes were used by the researchers. Participants were allowed to share their thoughts and emotions freely. Participants were appreciated and congratulated for coping with the pandemic and lockdown. Attempts were made to normalise their experience of anxiety, stress and other negative emotional states. They were allowed to make supportive comments to each other. They were encouraged to seek professional help, in case they observed drastic changes in their mental health. Post the discussion, participants were emailed psychoeducative material, self-care techniques and details of professional mental health services they can avail, if needed.

\section{Demographic Details}

After the participants had consented to be a part of the research, they were provided with a demographic sheet via social media. The demographic details from their completed sheets were collated. The participants were residing in NewDelhi and NCR $(N=3)$, Mumbai $(\mathrm{N}=3)$, Bengaluru $(\mathrm{N}=3)$, Andhra Pradesh $(N=1)$, Gujrat $(\mathrm{N}=1)$ and Bhopal $(\mathrm{N}=1)$. Out of 12 participants, 10 participants were working virtually from home and 2 participants were regularly travelling to their workspace. The educational qualifications of participants were undergraduate $(N=4)$, post graduate $(N=7)$ and doctorate $(N=1)$. Out of 12 participants, 7 participants were females and 5 were males.

\section{Results and Data Analysis}

The data that emerged from three FGD was interpreted using thematic analysis method. Data transcript was coded and categorised into miscellaneous themes. A thematic model was formulated to organise the relationships between themes, as following:

Using the above thematic model (Fig. 1) obtained, analysis and definition of individual themes and their codes will be discussed as following:

\section{Living Alone before Lockdown}

Participants reported that some of them willingly resided alone even before the lockdown while others said that due to uncontrollable circumstances they were stuck alone when the lockdown was announced. For some of them before lockdown, their life was stressful, unhealthy and replete with addictions. Some of these participants were also used to working from home. A participant shared that he chose to not move back with his family because he finds relationships with them toxic. 
Fig. 1 Thematic model depicting the networks of association among derived themes in analysis

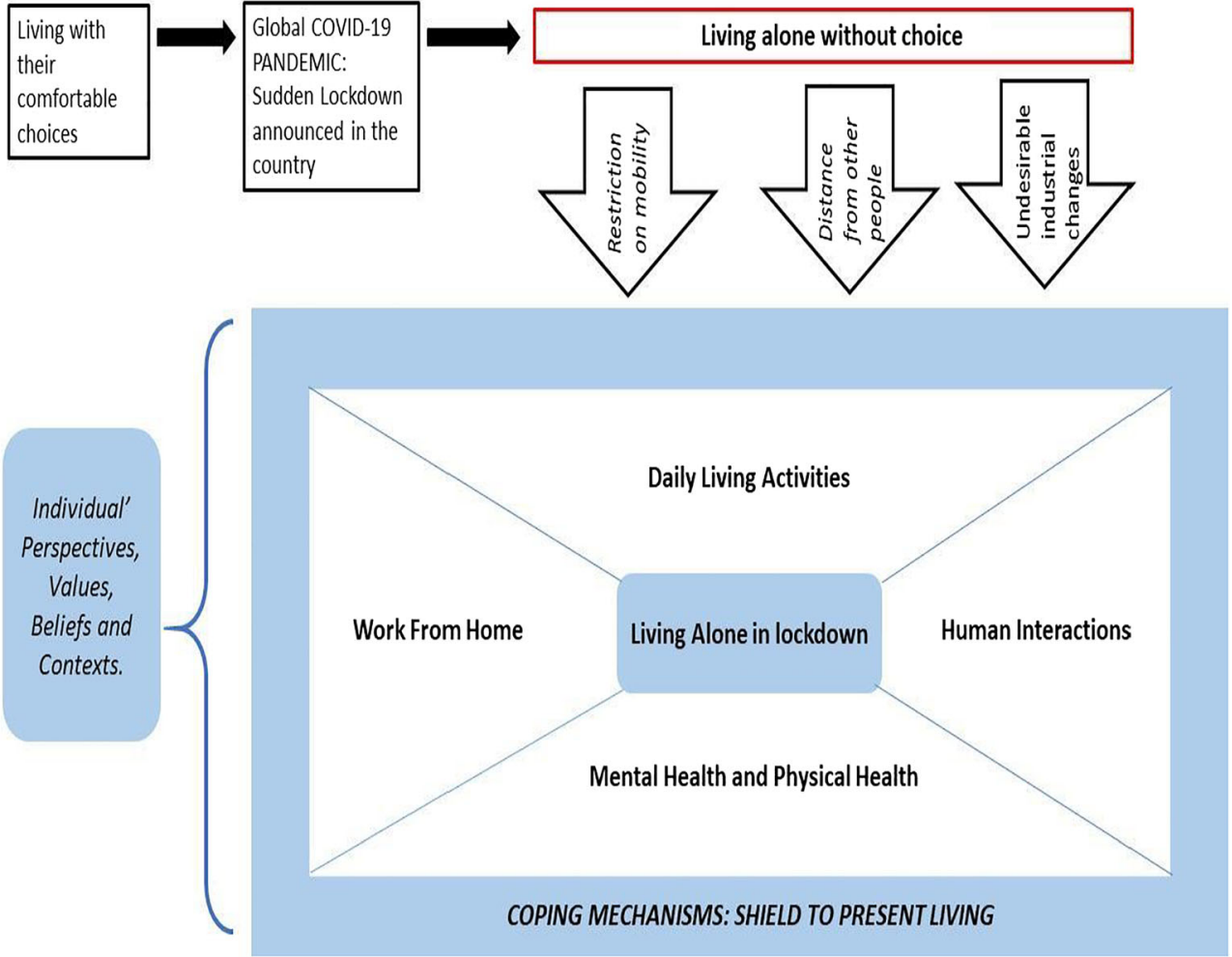

\section{Meaning Making of their Life in Lockdown}

Participants displayed their unique take on their lives post the lockdown. Their perspective ranged from a reframing of the lockdown as keeping oneself safe, referring to lockdown as a one-time lifetime experience, to losing track of time and the uncertainty of getting back to their usual lives. There was a belief that lockdown is a time and opportunity for working on oneself and achieving a direction and consistency with life while keeping mental health intact. They also added that it's a space to fulfil the complaints present with time in our usual life. Some also mentioned that they perceive a norm of being productive, learning new skills and acing work all around this lockdown.

“...I think that lot of us are trying to be productive because we are seeing all this productivity happening around as people posting things that probably cooking like specials, somebody is just working out so much. so I think there is a lot going out..."

Some also shared this is a space to develop positive health related habits and different hobbies. On other hand, some participants mentioned that lockdown is a threat that they live with on an everyday basis, which makes it difficult to motivate oneself for the activities required. Adding to the same, participant mentioned Sundays are hardly remembered and there is only one world: your home.

\section{Daily Activities and Living}

Lockdown affected the daily activities and living of participants, in multiple aspects.

To Schedule or Not to Schedule Some participants believed in making a schedule each day with all their daily activities and work, particularly a schedule that resembled their schedule before lockdown, aided their daily living at present. On the other hand, some believed a daily schedule or routine is not necessary, and life can be lived with one's flow and comfort.

The Scourge of Lockdown Due to the lockdown, the participants' daily routine was disrupted, specifically restriction of physical movement and recreational activities. Grocery shopping was difficult in the lockdown as they had to purchase and wash them all alone.

"...one thing that has really changed for me is the grocery shopping is that where I hate the lockdown and the virus being around. because that there is this ritual now related to it which is very weird also like I have to go buy all the groceries for myself for the week and I just come back and wash them, and it's very exhausting and tiring because I am living alone..." 
One of the participants specified a compulsion to hoard groceries. With work and daily activities, there was negligible time for self and hobbies and thus, it had become a very mechanical living. The confluence of physical boundaries of different living conditions like work and home had affected the balance between the two. One of the participants added that only Sundays were the days left of their choice to pursue new learnings or activities. Motivating themselves to complete all this work by themselves, was a task, exacerbated by the absence of roommates and domestic help. Disturbance with sleep cycle and appetite was another characteristic of their new life.

The Other Side of the Coin However, not all participants faced these challenges. Living alone in lockdown conceded a liberal space for performing daily activities and work, as well as for building positive health related behaviours, like exercise and healthy eating.

"...I have been in the most healthy, physically the most healthier mentally more healthier than I have been in years..."

Some of them found household chores easy, absence of flatmates meant it was easier to manage the house without them and their astute organization skills came handy. Consequently, some of them felt a sense of independence. Some positives about this lockdown included reduction in daily expenditure, eschewing traffic, learning new skills and consistent health related behaviours.

“..last 5 years was very stressful it was working 10 to 12 hours of work and lot of travelling and I stay in Bangalore so it would also include dealing with traffic.

I Kind of experienced peace..”

One of the participants added missing daily activity like driving and another participant added missing family.

Making Lemonade of the Lemons To manage the challenges, a few participants scheduled some chores for the weekend, others adopted strategies like cooking for two meals or even all three meals at the same time. Some of the participants were able to have balance and comfort with their living, consisting of cooking, working, reading, some outside walk and talking to the neighbours around.

\section{Working from Home}

Only individuals who are working were included in this research so most of them had to work from home because of the lockdown (Fig. 2). Though a few participants mentioned that due to the nature of their work, they could no longer work. This further created financial stress and career uncertainty for them. For some participants, their mode of working was virtual even before the lockdown, while others had to shift to a virtual mode post the lockdown. Some participants didn't have work initially because their organizations were in the process of transitioning to a virtual medium.

The Vicissitudes of Work from Home Life Many participants reported that their workload had increased by manifolds, their procrastination tendencies had increased affecting their productivity, video calls got exhausting, managing deadlines and working under extensive supervision was difficult and, important time like coffee breaks, and lunchtime could no longer bring the much-needed respite. The blurring of boundaries between personal and professional lives had created one world with several aspects which made their living difficult, particularly managing both household chores and work assignments.
“...it changes on the aspect that I used to come back home and do all of these things, but right now the thing is I am always at it so that is the part that has changed. I am although starting to lose track of time sometimes and I don't really know that how can we further manage it. because right now previously what used to happen was not about habit. I used to think of any task with a differ- ent allotted time to it. I would know when to do and what to do..... right now, it's like staying in one single space and then dealing with so much of excess pressure at workspace because I really want to tell you it has increase by many folds so then after that when I am doing all of these things it sort of becoming mechanical.."

One participant called work from home a sham. Another pointed out that there is a significant difference in working from home in a pandemic situation and in working from home otherwise because choice makes a huge difference as coerced working from home, decimates productivity and quality of work. Moreover, some of the participants added that they miss the freedom and non-virtual meetings at work.

Benefits of Working Remotely Participants who viewed the work from home situation positively shared that focus and efficiency (of both household chores and work) had improved thanks to less distraction caused by causal interactions with colleagues, and flexible work timings were appreciated. One of the participants also mentioned that he had slowly started working at his own pace, irrespective of the supervision and demands. One participant normalised procrastination and delay of work in pandemic. 


\section{Social Interactions and Relationships}

During the lockdown, two types of interactions were possible: virtual and non-virtual.

The Reassuring Physical Presence of Others Some participants had no non-virtual interactions while others interacted with neighbours, colleagues or vendors. They had observed a stark change in reality so the actual presence of people was reassuring. Their interactions with colleagues were professional and informative. One participant elaborated that via his neighbours, he was apprised of the myriad worries plaguing people such as the disruption of the market for businesspersons, the uncertainty of academic futures for college students and the constant presence of all family members for homemakers. Another participant happily shared that her neighbours helped her with groceries and food, besides participating in recreational activities with her.

"So when I go to the grocery store, when I go out to buy groceries, that time it's a little weird because you are seeing people, like it is a post-apocalyptic world where everyone is wearing masks and everyone is scared of everyone, so that is the only interaction I have. This is not an interaction but you know you look at people, you still like feel that there is at least people there in the world."

Alone and Still in Touch with People Most participants reported that virtual interactions had increased, along with the use of social media and of video calls, which were especially preferred for family and friends. Professional virtual interactions were for training and collaborating. With family, conversation topics centred on concern for health and future in the pandemic, and friends had been supportive virtually. There was a disagreement among the group about Covid-19 as a conversation topic. Although it was not a favourable topic, some were able to avoid it while others were bewildered how conversations without the same were possible. Due to shared existential threat, more initiatives or increased availability of time, increased connections were forged, contingent on proximity and possibility. They were able to connect with people they had not been in touch with since some time. Some had experienced that the frustration in their relationships had now made way for a safe space where they could share their thoughts and feelings. One participant shared that her parents had been insisting that she peruse matrimonial sites and identify probable partners but she had managed to delay that process. However, she said she was finally heeding their advice. Expression of care and concern had become more frequent in their interactions. They used metaphors of computer game and sci-fi movies to explain their virtual interactions and relationships. However, one participant drew attention to the fact that it was uncertain whether these interactions will continue post lockdown. The participants were divided in their stance on the changing nature of their personal and professional relationships.

A Sea without Salt Only thing missing was the physical touch, and hence it had gained a new found significance in their lives. The absence of social life and non-virtual human interactions affected most of them. They mentioned that the presence of human and emotional support would have eased the process of the living in this lockdown. Some of them shared that daily conversations or frequent video calls added to fatigue. One participant mentioned that the heightened workload had taken a toll on his personal relationships. One participant added that his relationship with his family had become tense due to concern over the lockdown.

\section{Mental Health}

For some participants, there were events other than Covid-19 and lockdown, which were stressful. One participant shared that he had recently gone through a break up while another shared that she had a conflict with a friend, which had persisted. One of the participants also had substance withdrawal and was very apprehensive about this process initially in lockdown.

"there are so many things that are happening subsequently in 2020, which are horrifying as well. So its like everyday the news is also filled with so many things so its not just that, the entire idea of 2020 being a very screwed up year."

Restrictions and Solitude Due to the restrictions on their physical movement and mobility, there was no respite from work via recreation or non-virtual connection with others. They could not reach out to others when they felt low. They missed their family, going out, driving and non-virtual meetings. Therefore, their initial reaction to the lockdown was panic, fear of Corona spread and sadness at feeling stuck.

"..with this kind of workload and all, yes it is taking....little bit of toll in terms of the enthusiasm that I used to feel before going to job in the normal days... Now I easily get bored or sometimes... it's the constant requests are coming and things are not getting done in a proper way and I have to like, even after giving the work to someone, I have to look into it again and correct it 
again and again so that makes me more irritated, earlier it not used to happen."

Effect on the Biological Cycle They observed a change in their sleeping patterns, including sleeping for longer hours. According to them, the blurring of boundaries between work and rest was to blame. Owing to reduced physical activity, their appetite was effected, which was linked to a change in their eating patterns.

Stream of Thoughts and Feelings One participant confided that after the lockdown was extended, he often wondered that his coping capabilities will not last for so long. They had become more emotionally vulnerable. There were a range of emotions they were experiencing: sadness, anxiety, loneliness, irritation, confusion, nervousness, shock and fatigue. They felt low on off days and observed a dip in their motivation as well as enthusiasm. They felt that they were just on the edge of failing. On days they couldn't be productive, they felt a loss of achievement. Some of them experienced panic attacks. One participant said that counting days since the lockdown created more anxiety.

\begin{abstract}
"..there is one more thing that's actually keeps coming to me. I hope that does not happen but I hear lot of news that many people... unfortunately lost their loved ones in this situation and they have not been able to say their last good bye to them so... I know nothing will happen to my loved ones but if it does, that's something that's worrying me. That if it does, I might not be able to visit in my hometown in that time and you know what I am trying to say."
\end{abstract}

Silver Lining Not everyone reported experiencing difficult emotions. Some participants felt positive, optimistic and calm as well as happy about making healthy choices and following a routine. One participant said that they were in a better state now. Technology was helping to deal with the feeling of loneliness. Perspective matters because some believed that vision of a better post lockdown world was just wishful thinking while others contemplated the scope for introspection, value for life and change in lifestyle.

\section{Coping Mechanisms}

With the unprecedented change in their lifestyle and environment, coping mechanisms were explored to arrive at an improved understanding of the present.
Time Heals Most of the participants mentioned that the initial couple of weeks were difficult, and slowly they started adopting or using different strategies than usual for a better living. For some this process and transition was relatively easy, some have found the ways to cope and some are still in the process of finding ways to cope. Their aim of coping was to ensure the possibility of having a positive mental and physical health.

Combating Stress with Structure Devising a new schedule inclusive of long sought activities like yoga, catching up with friends and relatives, reading and reaching out to friends and some time for self introspection and self talk were their ways to ameliorate the present. For some of the participants who had access to nature, nature was helping them to cope. Using the strength to push self on a daily basis only helped cope better. When they felt exhaustive and strained, they would give themselves a space to recover from it. For some of the participants household activities like washing clothes, utensils were brainless, so they supplemented these with conversations with loved ones or music. To cope with lack of physical activity, they tried to reduce their food consumption or incorporate physical exercise. Some amount of physical exercise helped them cope better with other activities of the day. One of the participants mentioned walking out on the terrace and being with his cats helped cope with the present. Setting small goals and priorities helped in planning the day with better distribution of energies. Another participant added that setting goals with their own capacity helped them feel accomplished and satisfied.

The Power of Thoughts Some coping mantras/positive affirmations included living one day at a time and seeing everyday as one day of lockdown instead of a number of days of lockdown. One of the participants shared their belief that once you begin a task, the mind helps in completing it. Doing a little bit everyday helped to cope. Not controlling things that are not in their hand, and only focusing on what they can control scaffolded acceptance of their situation. Another participant added that believing in the existence of bad days and good days helped cope better. Not being part of a productive race outside and just comparing with yesterday's own progress augmented coping. Another participant added a better post lockdown wishful thinking brings hope.

"It will totally depend on the person, how you take it. You can take it in a positive way, in near future thing things are going to be positive for you. If you are taking it in a negative way, things are going to be negative." 
Regulating Engagement with Outside World Most of the participants mentioned avoiding listening to the news constantly and just focusing on facts from reliable sources reduced their fear and anxiety around spread of coronavirus. Some of the participants added using avoidance of rumination to prevent recurrent negative thoughts and feelings.

Adoption of Feel Good Activities Learning new skills and practicing interesting activities, with small achievements was well as exploring their long sought hobbies were helpful, and positive impact of performing and pushing the day through other daily activities. Using substances like cannabis was reported as another feel good activity. One of the participants had substance withdrawal a month before lockdown and was initially apprehensive, but mentioned coping well with developing consistent practice of health-related habits such as daily physical activity and proper appetite.

"..thanks to the God that today we live in an era where we have the support of technology so we can interact with whomsoever you wish to, even though they are not living around you, you know you can watch movies or other stuffs so that is what is helping me throughout these days."

For some participants, lockdown itself came out to be a very calm and relaxing space from the exhaustive life before lockdown. Lockdown helped in relaxation and self-introspection, which was missing before.

Few of the participants mentioned, presence of emotional support could have helped them cope better, like having someone in their environment to feel secure and supported. Another participant added a space to go out and feel some fresh air, which can help cope with being stuck in four walls.

On the basis of the thematic analysis of participants' narratives, two noteworthy elements can be highlighted:

\section{Paradoxical Role of Technology}

Technology was beneficial to participants in two primary ways: it was a crutch to their solitude and it ensured that they maintained a stable income via virtual work. However, even if it allowed them to sustain and make human connections, it was not the same as non-virtual connections. The participants emphasised that physical touch was missing. Increased connection did not necessarily translate into increased closeness. Plus, the longevity of these connections could be questioned. In a similar vein, though work from home sustained livelihood of participants, it was the source of colossal stress and blurring of boundaries.

\section{Sources of Subjectivity}

A common theme between all participants was that they were young working adults living alone in a lockdown in India. The diversity in the data could be traced to innumerable other individual differences. Yet, there are two major factors that can be useful to consider: their context and their worldview. Their context helps us to fathom what aspects of the lockdown were novel, and thus, relatively less easy for them to manage. For instance, participants who even before the lockdown were living alone, worked virtually or knew how to cook, differed in their perception of and management of their life post lockdown. Another essential contextual factor could be how helpful their circumstances happened to be. The participants who had lost their jobs because of the lockdown or who had to work with increased workload undeniably had unique experiences of the lockdown. Secondly, their worldview and personal coping style mattered. Even if they were in the same river, they rowed their boats differently. Their personal beliefs and values contributed to the meaning they made of their experience- whether they construed lockdown as an opportunity for introspection or found their solitude a quicksand they struggled against.

\section{Discussion}

This research aimed to explore the lives of young working adults who were residing away from their family and friends during a lockdown in metropolitan cities of India. For this purpose, three focus group discussions were conducted with over 12 participants, and their narratives were analysed. This analysis bequeathed findings that were congruent to existing literature and some new territories which have not been explored by previous research. The disruption of daily routine, disorientation to time, restriction of personal freedom, changes in sleep cycle, fatigue caused by virtual work from home, increased digital use, worry about relatives falling sick and longing to meet family, anxiety, sadness, and receiving increased support from friends and family shared by participants, is supported by the literature review (Cellini et al., 2020, Flesia et al., 2020, Zhang \& Ma, 2020). However, contrary to literature review, participants cited events other than the pandemic that were stressful. They said that there were a number of unpleasant events broadcasted via news channels that evoked distress. In addition to that, studies have illustrated that solitude during the lockdown was a concern for some while others rekindled their bonds with their family and friends (Peshave \& Peshave, 2020; Abel \& McQueen, 2020). However, the present study's participants shared their experience of continued conflict with their family or friends despite the increased distance created by the lockdown and raised the pertinent question of the sustainability of rekindled 
bonds post lockdown. In fact, participants shared that frequent digital connection with family or friends was actually giving rise to fatigue in the long term, even though technology was the only thing keeping them tethered to that social life. Though it was observed that work from home created fatigue, participants didn't forget to applaud this mode for its benefits.

Most participants shared that the uncertainty surrounding the pandemic, lockdown and their futures was a cause of worry, stress or anxiety. Research does substantiate that ambiguity of a situation can make stress appraisals more likely, along with uncontrollability of the stressor (Taylor, 2015). Participants' degree of control over the pandemic, lockdown or their futures was considerably low.

Unlike coping mechanisms suggested by researchers such as Hiremath et al. (2020), the participants of the present study shared their unique strategies of dealing with the pandemic. The mental health of participants and their coping approaches to it can be understood from the lens of death anxiety as well. Irvin Yalom conceptualised that death is a source of anxiety for most of us (Cooper, 2016). He explained that to avoid that anxiety, we try to live our lives as if we are never going to die, that is, we use sundry defences to protect ourselves from this anxiety. However, he adds that some of us have boundary experiences which compel us to confront the notion of death, such as being diagnosed with a terminal illness or witnessing the death of a loved one. Covid-19 is not necessarily a terminal illness. Statistics show that an enormous number of people have recovered from the virus (WHO,2020). However, the possibility of dying from the virus has been substantial. None of the participants directly and openly mentioned death anxiety in any way but they did voice their concerns about the health of their loved ones. They mentioned that they feared that their loved ones might be infected with the virus. They articulated their desire to one day be able to go out with the freedom they used to have, that is, without worrying about masks and sanitation and other precautions. This indicated that there was some fear or anxiety about them getting infected, considering their residence in red zones as declared by the government of India. Red zones were those areas in the country where the maximum number of Covid-19 cases were reported, thus calling for maximum restrictions in the lockdown. This implies that the fear of Covid spread was palpable in these regions and the participants also had to endure increased restrictions. Yalom further expounded that when confronted with notions of death, several individuals begin to acknowledge and accept their mortality, and consequently, begin to engage with the positive aspects of their life. Most of the participants have spoken about their increased connection with others and their engagement with hobbies, they did not have time for before. Trying to regulate their consumption of news or trying to have conversations that were not about Covid-19, could also be seen as ways of protecting oneself from death anxiety.
The picture of the impact of Covid-10 on mental health of the participants would be incomplete without the perspective of crisis theory. Caplan $(1964,1974)$ considers crisis is provoked when a person faces a problem for which $\mathrm{s} / \mathrm{he}$ appears not to have an immediate solution and that is for a time insurmountable through the utilization of usual methods of problem solving. Caplan suggested a period of upset and tension follows during which a person makes several attempts at the solution of the problem. Eventually some kind of adaptation and equilibrium is achieved which may leave the person in a better or worse condition prior to the crisis. Similarly, the present situation of living alone in unprecedented lockdown came as a crisis for the participants. Most of the participants mentioned that it took initial few weeks for them to devise the new coping mechanisms which were more helpful. Caplan also suggested that the essential factor determining the occurrence of a crisis is an imbalance between the perceived difficulty and importance of threatening situations and the resources immediately available to deal with it; crisis refers to the person's emotional reaction, not to the threatening situation itself. Similarly, in the present study living in lockdown was more difficult and threatening on a scale of intensity compared to the COVID -19 outside as reported by participants in discussion.

Caplan's crisis theory is grounded in the concept of homeostasis. According to him, the organism constantly endeavors to maintain a homeostatic balance with the outside environment. When this balance is threatened either by physiological or psychological forces, the individual engages in problem solving activities designed to restore this homeostatic balance. A crisis is considered an upset of a steady or homeostatic state. Similarly in the present situation, unprecedented created an effect in the homeostatic state of living for the participants. They mentioned struggling to achieve an equilibrium with their daily living, work from home and their mental and physical health.

Parad (1966) in acceptance of Caplan's model stresses the importance of an individual's perception of what constitutes a crisis. Such a perception difference was present in the present study, where this lockdown was a space for self-recovery and health maintenance and for some a period of deterioration in mental and physical health. Some of the participants mentioned lockdown as a time and opportunity for working on self and achieving a direction and consistency with life while keeping mental health intact. Some also perceived it as a space to develop positive health related habits and different hobbies. Whereas some participants perceived lockdown as a threat that they live with on an everyday basis, which makes it difficult to motivate oneself for the activities required.

Caplan (1964) also suggested four stages of crisis reaction. First, individuals are exposed to a crisis state. Second, followed with failures of prior coping mechanisms, leads the crisis to persist. Third, mobilization of resources and coping 
mechanisms ensues, in order to reduce the tension or attain homeostasis. Fourth, if a crisis persists, tension mounts beyond a further threshold resulting in major breakdown in the individual's mental and social functioning. In the present study, most of the participants were moving to and fro among the last two stages of finding new coping mechanisms and acute breakdowns. Several participants mentioned that initially the first two weeks of lockdown were more difficult and they were adapting with new coping mechanisms. For some the outcomes were positive with better living and mental health, whereas for some, it was deteriorating especially mental health.

Another lens that is imperative to adopt while we try to understand the participants' narratives is that the suddenness of the lockdown was not the only stress provoking aspect, the participants were living alone during this time. Though existing literature discusses the essence of social support and the consequences of its absence, the circumstances of the participants were unique because they were not in solitary confinement due to criminal persecution, there was no disruption of necessary infrastructure typical of physical disasters, they were not infected with any illness that isolated from others and they had not lost touch with their social network entirely. Rami (2013) along with several researchers have highlighted the effect of social interactions and support in crisis and negative experiences. Most of the participants' social interactions and social support were virtual. Several participants shared the positive growth in their interactions and social support. Though there was consistent social support and interactions present virtually, most of the participants mentioned that the physical presence of human and social support would have been a better support. Turner (1981) also mentioned the importance of social support around supporting emotional wellbeing in difficult situations, similarly the participants felt such need while coping with the above crisis.

Since all the participants were working at the time, work from home was a crucial factor that defined their experience during the lockdown. Boundary and Border theory (Ashforth, Kreiner, \& Fugate, 2000; Clark, 2000) conceptualizes the work and non-work interface in terms of boundaries or borders that surround the different life domains. They also added that individuals work towards establishing balance with demands and requirements of both the domains. The strategies used by individuals for the same works on a continuum from segmentation to integration. Similarly, with respect to work from home and daily living activities, participants of the present study had a mixed approach. Some of the participants were on the segmentation side and some on the integration side of the continuum. There was an initial struggle in identifying the same as there were no physical boundaries present compared to living before lockdown. Due to absence of physical boundaries, the fluctuations were quite often and were also based on the nature of their work. One of the participants working without a schedule with integration of several activities was more comfortable and successful in completing daily activities and work, whereas some participants with a schedule for daily activities and office work succeeded in coping with blur boundaries and also being productive.

There were a couple of protective factors moderating the above impact discussed. First, it is essential to contextualise the experiences of the participants in their digital privilege. The digital divide in India is drastic. Since participation in this research necessitated not just access but an ability to seamlessly use high speed internet services, it can be claimed that the participants unequivocally had sustaining connections with others. In addition to that, they had a job or possessed the skills to be hired for a job during the lockdown. Though we have made a distinction between the virtual and the non-virtual, there are scholars who proclaim that not only are our lives even more starkly defined by our reliance on technology in the time of the pandemic, we live in a post-digital world (Coeckelbergh, 2020). This implies that the dualist notion of online and offline is facile because they are not two different spheres at all.

There are theories and studies that have speculated about the impact of myriad crises, ranging from death to traumatic experiences, about the possibilities of modification in the boundaries of our life, about the impact of lack of physical presence people in our life but the pandemic situation was unique, despite sharing resemblances to what has already been conceptualised and explained. During the lockdown, the participants were unable to meet their loved ones but could remain digitally connected to them. A number of their daily activities had come to a grinding halt but their work continued. The backdrop to these experiences was a pandemic thousands of people were succumbing to daily.

\section{Conclusion}

The objective of the research was to map out the lived experience of young adults of living alone in a lockdown. With the help of focus group discussions, an attempt was made to unravel how these individuals managed their work life, their household and keeping in touch with people who mattered. The discussions brought into the spotlight the role of their life circumstances and worldview along with their coping styles in making sense of their experience. The discussions highlighted the effects on their mental health and 
changes in their usual coping mechanisms as a result of this unprecedented event.

\section{Limitations and Future Directions}

Since the context of the participant group was unique, results can't be generalized to all adult individuals, but they can be generalised to individuals situated in similar settings in other countries. Third world multicultural countries with high population density and a comparable trajectory of the spread of Covid-19 may be representative of settings that these results can be generalised to. Another limitation of the study was the lack of representativeness of the participant group because young adults were invited to be a part of this research via social media. Therefore, the authors could only reach a limited pool of individuals. The small number of participants can be a limitation, as well. Potential participants were informed that they will be required to share their experience of living in the lockdown, which may have led individuals who were severely distressed in the lockdown to not opt for this study. In the wake of the lockdown in India, there were several groups that were severely affected yet the scope of this paper is limited to working class individuals living in metropolitan areas alone, and with digital access. To comprehensively understand the plight and resilience of even this limited group, other relevant groups such as their family members, their friends and colleagues could have been included. Onfield study of their neighbourhood and workspaces could have added valuable snapshots to the big picture of their lives. Besides participants, there were methodological limitations as well. The focus group discussions were facilitated by a self constructed guide of questions. Though participants were encouraged to share narratives beyond those questions, it is possible that the researchers' line of inquiry influenced and reined in the discourse that emerged from the discussions. In addition to that, though the objective of the FGD was to enable a discussion among the group, participants may have felt inhibited to disclose details of their lives with the group.

This research highlights the need of holistic psychosocial evaluation and intervention for such vulnerable groups. Further research can look into the intervention approaches required to deal with the distress of such groups in such situations. Present paper also identifies some coping mechanisms which can be used to support such interventions. These can be useful for mental health professionals and for public policy makers. These findings can be an impetus for further research about long term crisis interventions, the changing nature of relationships in the absence of physical interaction, process of coping in solitude and the shift to a virtual workspace. This research could be a contribution in the field of positive psychology, in terms of the capacity of resilience of humans.

\section{Declarations}

Conflicts of Interest/Competing Interests On behalf of all authors, the corresponding author states that there is no conflict of interest.

\section{References}

Abel, T., \& McQueen, D. (2020). The COVID-19 pandemic calls for spatial distancing and social closeness: Not for social distancing. International Journal of Public Health, 65, 231.

Ashforth, B. E., Kreiner, G. E., \& Fugate, M. (2000). All in A Day's Work: Boundaries and Micro Role Transitions. The Academy of Management Review, 25, 472-91.

Barkur, G., Vibha, \& Kamath, G.B. (2020). Sentiment analysis of nationwide lockdown due to Covid-19 outbreak: Evidence from India. Asian Journal of Psychiatry.

Benerjee, D. (2020). Psychological preparedness for the Covid-19 pandemic, perspectives from India. Psychiatry Research, 288, 112999. https://doi.org/10.1016/j.psychres.2020.112999.

Caplan, C. (1964). Principles of preventive psychiatry. New York: Basic Books, Inc..

Caplan, C. (1974). Support systems and community mental health: Lectures on concept development. New York: Behavioral Publications.

Cellini, N., Canale, N., Mioni, G., \& Costa, S. (2020). Changes in sleep pattern, sense of time, and digital media use during Covid-19 lockdown in Italy. Journal of Sleep Research, 29.

Clark, S. (2000). Work/family border theory: a new theory of work/ family balance. Human Relations, 53(6), 747-770.

Clarke, V., \& Braun, V. (2013). Teaching thematic analysis: Overcoming challenges and developing strategies for effective learning. The Psychologist, 26(2), 120-123.

Coeckelbergh, M. (2020). The postdigital in pandemic times: A comment on the Covid-19 crisis and its political epistemologies. Postdigital Science and Education, 1-4.

Cooper, M. (2016). Existential therapies (2nd ed.). Thousand Oaks, CA: Sage.

De Sousa, A., Mohandas, E., \& Javed, A. (2020). Psychological interventions during COVID-19: Challenges for low and middle income countries. Asian Journal of Psychiatry, 102128.

Denzin, N. K., \& Lincoln, Y. S. (2017). The SAGE handbook of qualitative research. Sage Publications.

Flesia, L., Fietta, V., Colicino, E., Segatto, B., \& Monaro, M. (2020). Stable psychological traits predict perceived stress related to the COVID-19 outbreak.

Ganguly, D., Misra, S., \& Goli, S. (2020). India's COVID-19 Episode: Resilience, response, impact and lessons.

Goyal, K., Chauhan, P., Chhikara, K., Gupta, P., \& Singh, M. P. (2020). Fear of COVID 2019: First suicidal case in India! Asian Journal of Psychiatry, 49, 101989. https://doi.org/10.1016/j.ajp.2020.101989.

Hiremath, P., Suhas Kowshik, C. S., Manjunath, M., \& Shettar, M. (2020). COVID 19: Impact of lock-down on mental health and tips to overcome. Asian Journal of Psychiatry, 51, 102088. https://doi. org/10.1016/j.ajp.2020.102088.

Holmes, E. A., O'Connor, R. C., Perry, V. H., Tracey, I., Wessely, S., Arseneault, L., ... \& Ford, T. (2020). Multidisciplinary research priorities for the COVID-19 pandemic: A call for action for mental health science. The Lancet Psychiatry. 
Lancet, T. (2020). India under COVID-19 lockdown. Lancet (London, England), 395(10233), 1315.

Naguy, A., Moodliar-Rensburg, S., \& Alamiri, B. (2020). Coronaphobia and chronophobia-a psychiatric perspective. Asian Journal of Psychiatry, 51, 102050.

Parad, H. J. (1966). The use of time-limited crisis intervention in community mental health programming. Social Service Review, 40(3), 275-282.

Peshave, J., \& Peshave, M. (2020). Covid-19 lockdown-a blessing or curse ? CLIO An Annual Interdisciplinary Journal of History, 6(1), 537-544.

PM Modi announces a 21 day lockdown as Covid-19 toll touches 12. (2020, March) The Hindu. Retrieved from https://www.thehindu. $\mathrm{com} /$ news/national/pm-announces-21-day-lockdown-as-covid-19toll-touches-10/article31156691.ece

Rajkumar, R. P. (2020). COVID-19 and mental health: A review of the existing literature. Asian Journal of Psychiatry, 102066.

Rami, S. (2013). Social support, emotional well-being, and emotion regulation: A mediation model (Doctoral dissertation).
Taylor, S.E. (2015). Health psychology. McGraw-Hill Education.

Torales, J., Higgins, M., Castaldelli-Maia, J.M. \& Ventriglio, A. (2020). The outbreak of Covid-19 coronavirus and its impact on global mental health. International Journal of Social Psychiatry, 1-4, https://doi.org/10.1177/0020764020915212.

Turner, R. J. (1981). Social support as a contingency in psychological well-being. Journal of Health and Social Behavior, 22(4), 357-367. https://doi.org/10.2307/2136677.

World Health Organization. (2020). WHO Director-General's opening remarks at the media briefing on COVID-19-11 March 2020.

Zhang, Y., \& Ma, Z. F. (2020). Impact of the COVID-19 pandemic on mental health and quality of life among local residents in Liaoning Province, China: A cross-sectional study. International Journal of Environmental Research and Public Health, 17(7), 2381.

Publisher's Note Springer Nature remains neutral with regard to jurisdictional claims in published maps and institutional affiliations. 